\title{
Ontological Architecture for Management of Telemonitoring System and Alerts Detection
}

\author{
Amine Ahmed Benyahia, Amir Hajjam, Vincent Hilaire and Mohamed Hajjam
}

Additional information is available at the end of the chapter

http://dx.doi.org/10.5772/3158

\section{Introduction}

Telemonitoring (health monitoring, Home Health Care) is a branch of telemedicine that aims to restore independent living in their homes, to people suffering from various diseases and disabilities that would force them to a hospitalization or placement in specialized institutions (patients suffering from certain chronic diseases, disabilities, but also the frail elderly) [1].

This emerging area has led to much research during the past two decades and led to some development of systems that allow the maintenance to their homes of these people. To prevent the risks associated with lack of medical support "presential", technological systems must be implemented to provide graduated responses, adapted to individual cases, but it must in addition ensure respect of privacy of the person and not disrupt their lifestyle.

These systems must be open, able to integrate various technologies, at the same time flexible enough to adapt to the case of each patient, and to reflect changes in health state of a person.

Telemonitoring is based on the transmission and interpretation of medical indicators (clinical, radiological or biological) collected by the patient himself or by a health professional (doctor, nurse, etc) [2]. They can be interpreted by a health professional, or through programs and specialized software. The interpretation may lead to the decision to intervene with the patient or just provide advices.

Among the benefits of telemonitoring: The friendliness of the technology. A better understanding of the health status and better control of symptoms that give a sense of security. Economic system by minimizing hospitalizations and unnecessary displacement.

Telemonitoring is characterized by the use of vital data sensors necessary for diagnosis as blood pressure, weight, temperature, blood oxygen saturation, etc. These sensors are, in 
most cases, wireless (Bluetooth, Wi-Fi, etc) for more freedom of movement and portability [3]. Other sensors can be used as behavioral sensors (for example if the patient's spirit to climb stairs that affects blood pressure), or environmental sensors such as ambient temperature.

The smart house system can easily take advantage of existing technologies in home automation to facilitate the completion of certain tasks by the person, for example, unlock a door in an emergency, turn on or turn off lights, adjust the heating. Moreover, robots can help the person to perform certain daily tasks. Thus, a manipulator arm can be mounted on a wheelchair or on a mobile robot, to press a button, a lever handle door or catching. Also, depending on conditions, the system should take into account the therapeutic machines: syringe pump, infusion, dialysis machine or pill. It should be noted that these machines load their own sensors and can also participate in the task of data acquisition.

In earlier generations, the data collected were sent to physicians via the Internet to be interpreted, and thus detect anomalies and emergencies. With technological advancement, applications were developed to interpret and detect abnormal situations. These applications are implemented in the patient on a single computer, on touch pad or smart phone. If an anomaly is detected, these applications react accordingly, either by providing advice to the patient, or by calling an ambulance (depending on the level of the anomaly). The doctor in charge can track the state of his patients and receives alerts and anomalies detected.

In Part 2, we present generalities on ontologies and their advantages. Then in Section 3, we present some related work present in the literature, their advantages and inconveniences. Part 4 includes the proposal of a generic and adequate solution to the problem. Finally, we finish with a conclusion.

\section{Ontologies}

\subsection{Definition}

The first accepted definition for an ontology is that of [4], "explicit specification of a conceptualization".

This definition has also been recently clarified by [5] to be: "Ontology is an explicit formal specification and a shared conceptualization".

In this definition, it is necessary to correctly interpret each used term. Formal: the machine can understand. Explicit: the concepts, relationships, individuals and axioms are explicitly defined. Shared: knowledge representations are shared by a community. Conceptualization: abstract model of a part of the world that we want to represent.

\subsection{Dimensions of classification}

Ontologies can be classified along several dimensions. Among these, we focus on the typology based on the object of conceptualization. 
Ontologies can be classified according to their conceptualization object as follows: Top level ontology, Domain ontology, Task ontology, Application ontology. As shown in Figure 1.

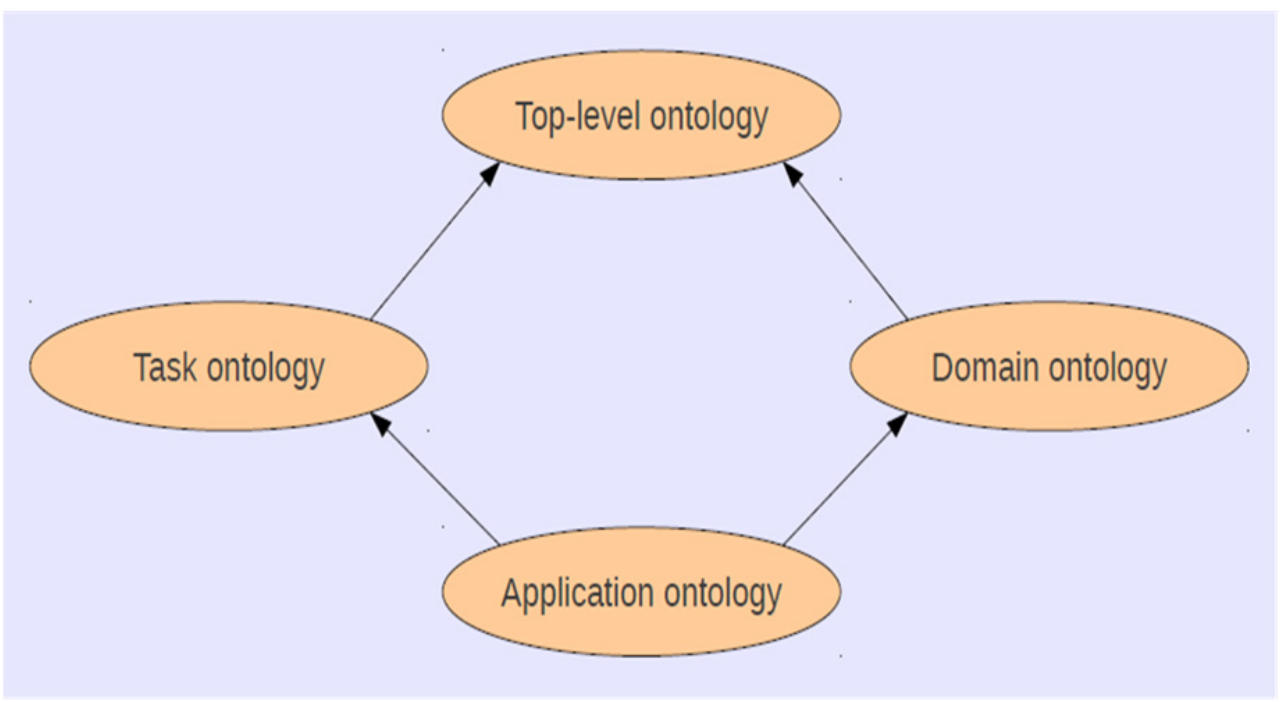

Figure 1. Classification of ontologies according to the object of conceptualization

Top level ontology [6], this type of ontology describes very general concepts or common sense knowledge such as space, time, event, action, etc. These concepts are independent of a problem or a particular area.

Domain ontology [7], this ontology governs a set of vocabularies and concepts describing an application domain or the target world. It characterizes the knowledge of the area where the task is performed. Most existing ontologies are domain ontologies.

Task ontology [7], this type of ontology is used to conceptualize specific tasks in systems. It governs a set of vocabularies and concepts describing a structure of performing the tasks domain-independent.

Application ontology [8], this ontology is the most specific. The concepts in the application ontology are very domain specific and particular application. In other words, the concepts often correspond to roles played by domain entities while performing a certain activity.

\subsection{Contribution of ontologies}

Ontologies provide a common semantics. This means that all individuals and concepts involved can be explicitly defined in terms of their relationships and attributes. Therefore, ontologies are interpreted by a machine and shared between several people. This facilitates and improves the quality of diagnosis and the process of decision support. Also, several people can work together without any ambiguity or loss of information. 
Ontologies provide a model of high level abstraction of daily workflow. This model can be adapted to each organization. In other words, any organization can have an ontology adapted to its particular situation.

Ontologies are generic and reusable. If it is necessary to build a big ontology, it would be possible to integrate several existing ontologies describing portions of a field. Top-level ontology can also be reused and extended to describe a specific area of interest.

Ontologies are very easy to maintain and with very minimal cost.

\section{Related work}

There are a lot of works related to our study in the literature. We chose to present the most important dividing them into two groups according to their use of ontologies.

\subsection{Systems that do not use ontologies}

[9] worked on a study of home telemonitoring for the elderly (applied to Alzheimer's disease). Their system allows the detection of nycthemeral rhythms drifts from location data (they use location sensors to see where the patient has spent his time during the day). Recordings are made every second. Then one day measures are grouped by period of one hour or 15 minutes, to be compared by Hamming measure. Through this comparison, they can know when the patient slept and how many hours of sleep he had.

Other studies in the same context as [10-12] were made using sensors location of the patient. These studies are based on classification methods to analyze the patient's daily activities.

[13] propose a telemonitoring system with architecture of three levels: the sensor network, the patient server that collects and transmits the information, and the hospital server that processes information and makes them available to physicians. This system uses data mining to detect anomalies.

[14] worked on the project TISSAD which develops medical remote monitoring systems at home. These systems are designed for elderly and / or chronic conditions, to prevent accidents or aggravation of their health conditions. The system is based on the recovery of vital or behavioral data and saves them in a database. Then due to an intelligent module, it makes computer-aided diagnosis. The project TISSAD was centered on the user by consolidating data into four classes: identification, historical requirements, medical history and medical data.

[15] proposed a strategy to implement an alarm component in remote monitoring system of elderly people. The system consists of units in the homes of people monitored for collecting from sensors (medical and environmental) and sending data, and a call center with a server for recording and tracking data. Alerts are modeled in XML. 


\subsection{Limits of systems that do not use ontologies}

Systems seen previously are based on old technologies. These technologies are characterized by the absence of semantics, which implies that the machine is not able to interpret the results. The lack of ontologies reduces their performance and making them difficult to share and evolve. In addition, they use data formats specific to them, making the information and data not generic and communication with other systems very complicated. The decision support is either lacking or based on data mining, or by using very complex processes.

For systems that do not provide decision support as those presented by [9, 12], despite that its systems are simple and not expensive, the machine cannot interpret data collected after their classification, specialists must monitor the activities of patients.

Using only data mining, the system will generate false alarms or will not detect anomalies due to the probabilistic characteristic of data mining. Errors will therefore introduce the facts base and will generate even more mistakes. This implies a degradation of system performance over time.

Without the use of ontologies, the inference process becomes very complex and difficult to change because the handled information is poorly structured so difficult to exploit. Also due to the characteristic "linked data" of ontologies, inference becomes easy and automatic as the use of transitivity, reflexivity, etc.

\subsection{Systems using ontologies}

[16] propose a system of decision support for remote monitoring of patients with heart failure. The system is based on an ontology which includes patient data: posture, pulse sensor, physical activities and alerts. The decision aid is based on inference using rules managed by an inference algorithm.

This system does not take into account physiological measures that are connected to the heart failure like blood pressure and weight data, nor the patient's environment such as temperature and humidity. Moreover, the context of the patient is very small for such physical activity as it contains statements: run, walk or anything, and also the posture he has only two states: lying or standing. Finally, the proposed inference algorithm is not optimal because a rule can have multiple conditions, for each condition the algorithm cover all the facts base, when he could have in some cases use the results of other conditions.

[17] propose a system based on multiple ontologies: the personal data of the patient, the context of the house (environment), social context (people connected to the patient) and alarms. The system uses the rule-based inference of first order, these rules are very dependent on the parameters (example: if temperature $>40$ then alert) making them non generic and non-scalable. The rules do not take into account changes in health state of the patient. Moreover, the rules can change from one patient to another but it is done manually by experts implying important feedback. 
[18] detail the design and implementation of a platform for reasoning to anticipate and react intelligently in situations demanding long-distance care or at home. The system manages intelligent agents, whose behavior is defined and validated by ontologies and rules. A development methodology has been adapted to support the process of knowledge acquisition. Log files are stored in XML to make data mining algorithms.

[19-21] proposed an ontological architecture for modeling a system Smart Home e-health. The goal is to provide an adaptive system for extending the home support of an aging person with diminished cognitive autonomy. The architecture is based on seven ontologies: housing, equipment, person, behavior, task, software application and the decision. These ontologies are interconnected.

\subsection{Limits of presented systems using ontologies}

These systems only partially integrate ontologies and do not contain domain ontologies that provide a controlled vocabulary standard to better share information and make it more generic.

In most cases, information about the patient are not fully exploited, each system is based on part of data (physiological, environmental, behavioral), although these data are strongly related and should be exploited in the same process to ensure decision support more accurate.

Moreover, the inference engines are based on rules defined by experts, but these rules are not scalable and do not take into account changes in health status of the patient. In the case where experts fail to mention a few cases and few rules, the system is unable to detect anomalies.

[22] Propose the most complete architecture in those studied. It consists of several ontologies for symptoms, diseases, medications, measures and patients. An inference engine on these ontologies. In addition, a status history of the patient and a history of actions taken are stored in another database. Methods of data mining are used on both historical to produce new rules that will supply a second inference engine. This inference engine has as facts base both historical.

This system is very complicated because it must handle ontologies and relational databases at the same time. This implies synchronization between the two bases, with a risk of loss of information and semantics. The use of two inference engines simultaneously reduces system performance in terms of response time.

\section{Proposition}

From systems seen previously, we will try to propose a general and generic architecture based on ontologies for telemonitoring of elderly person or person suffering from a chronic disease such as heart failure, kidney disease or Alzheimer's pathology. 
This architecture is based on ontologies and decision support. The aim is to detect anomalies or dangerous situations by collecting physiological data (heart rhythms, temperature, weight, etc.) or behavioral (daily activities, posture, etc) about the patient as well as information from its environment (light, temperature, humidity, magnetic field, etc).

This architecture is shown in figure 2 and consists of several parts with two principal actors who are medical experts and system experts. The architecture contains two types of ontologies:

- The ontology that manages the system (users, sensors, measurements, alarms, etc). We call this ontology "application ontology". The architecture of this ontology is defined by the system specifications.

- The domain ontologies are used to define a controlled vocabulary (diseases, medications, symptoms, etc). These ontologies are connected to the application ontology and will complete it by defining some of its concepts. These ontologies are built by medical experts.

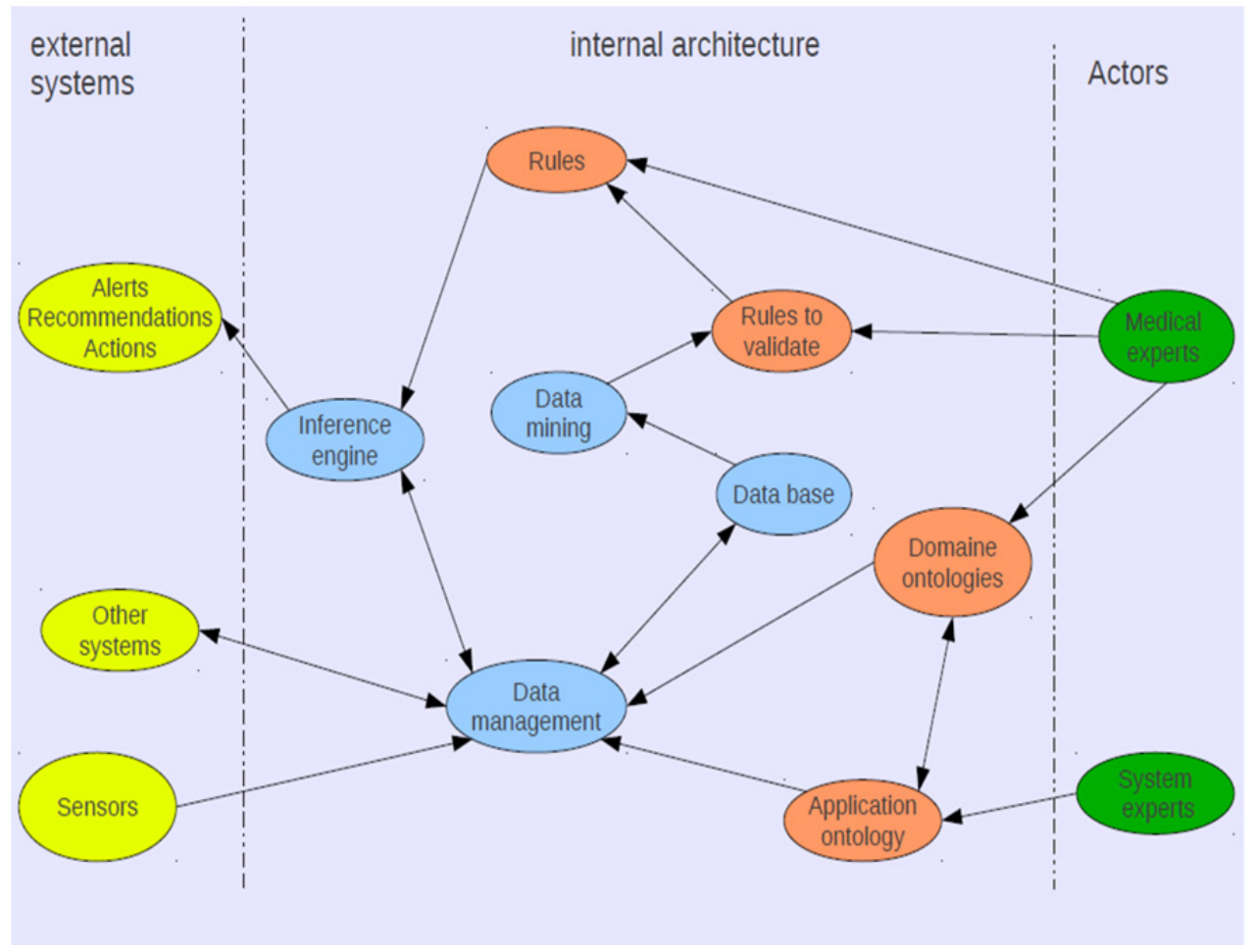

Figure 2. System Architecture

The decision support is based on an inference engine that receives as input the rules of inference and the facts that is provided by the "data management" part. The rules are introduced either by medical experts, or generated by a data mining then validated by medical experts. The inference engine detects anomalies and raises alerts informing doctors. 
- Data Management part is the core of the system, its main features are:

- Receive data from sensors.

- Communicate with other systems.

- $\quad$ Provide a facts base for the inference engine and receive the data inferred.

- Manage the database and ontologies.

- Our system must meet the following requirements:

- It must cover all patient information (physiological, environmental, behavioral), and represent them to facilitate the detection of abnormalities and dangerous situation.

- It must contain a controlled vocabulary to communicate unambiguously with other systems.

- The solution should be generic and can be used for any system of medical monitoring.

- Collect patient data from sensors or doctors and store them.

- Infer the state of the patient based on his background and the data collected and the rules defined by medical experts.

- A data mining to generate new evolutives rules.

- Rules must be generic, and evolve with the patient's condition.

- Detect anomalies and issue warnings to physicians and recommendations for the patient.

\subsection{Application ontology}

The application ontology is the heart of the system. It describes the various components of the system (users, sensors, measurements captured, the input data, alerts generated, etc). This ontology also defines the tasks of different actors in the system.

This ontology should contain different types of users, patients, medical users (doctors, nurses, etc.) that are assigned to patients. Other actors may have to use our system as the patient's relatives (families and neighbors), technicians who manage the technical aspects of the habitat or response teams (SAMU, firemen, call center emergency, etc.).

This ontology should describe the care habitat of patient (home, hospital, etc.). These habitats include technical equipment (different types of sensors, actuators and machines), or non-technical that the patient uses daily (furniture, dishes, etc.).

The application ontology is patient-centered. The data collected about the patient must be stored in this ontology to be used in the process of detecting anomalies in the health status of the patient. The collected data can be physiologies data, behavioral data or environmental data. This data is collected by the sensors that are suitable, which are part of technical equipment.

\subsection{Domain ontologies}

The domain ontologies aim to complete the application ontology by providing a controlled vocabulary, as for diseases, symptoms and medications. These ontologies can provide a language for sharing and communication between different actors in the system. With the 
semantics of these ontologies, our system can interact easily with other systems. Thus, data and information processed are generic and can be introduced into other systems.

These ontologies are built by medical experts, aided by knowledge engineers to formalize them. These ontologies can be linked between them, for example, link disease to symptoms.

We can also construct ontology from a medical corpus made up of stories, publications, regulations, etc. With text mining techniques applied on this corpus, we define a controlled vocabulary. This controlled vocabulary is transformed into ontology by creating relationships between different terms such as the relationship of hierarchy.

We can also use controlled vocabularies existing in literature and transform them into ontologies. [23] use this method to build two ontologies, one for diseases and the second for the symptoms.

Due to the reusability of ontologies, ontologies existing in literature can be reused as the one built by $[23,24]$.

\subsection{Decision support}

The decision support is reflected in the detection of abnormalities and changes in health status of the patient. It is ensured by an inference engine based on rules. These rules should be as generic as possible, and must evolve with the patient's state.

Inferred data provide recommendations and advice to the patient if necessary. If abnormal conditions are detected, in this case, the system must send alerts to the medical staff or the patient's family or his neighbors, or in an emergency, the call center and teams intervention. They can also lead to an action on the machines and actuators in the habitat. Inferred data can help the doctor diagnose the patient by providing information of the evolution of his health.

The rules are defined by medical experts and can change over time. Rules can also be generated after a data mining done on the databases. As data mining is probabilistic, rules must be validated by medical experts.

For each new event (data collection), the inference engine is started to try to apply the rules of this new data. If new data is generated by the inference engine, then resume the process from start, taking the new data as new events. This process stops when there are no rules to fire or that rules do not generate new knowledge.

\section{Conclusion}

In this paper we proposed a solution for managing data and detect anomalies in a telemonitoring system.

Telemonitoring systems are based on the collection of information from physiologies, environmental or behavioral sensors. In the first systems, these data were interpreted by 
physicians directly. But with technological advancement, expert systems have been developed for the automatic processing of data.

We studied various proposals and solutions existing in literature and we presented the most important.

We proposed a general solution that relies on the use of different types of ontologies, an ontology management system, and domain ontologies.

Using ontologies, this solution is generic, sharable and can communicate with other systems without ambiguities.

This solution is based on inference to detect anomalies and changes in patient's state. This inference is based on rules that are defined by medical experts or generated by a data mining followed by a validation by medical experts.

\section{Author details}

Amine Ahmed Benyahia

Université de Technologie Belfort-Montbéliard, Belfort, France

NEWEL, Mulhouse, France

Amir Hajjam and Vincent Hilaire

Université de Technologie Belfort-Montbéliard, Belfort, France

Mohamed Hajjam

NEWEL, Mulhouse, France

\section{References}

[1] Pierre Barralon. 2005. Classification et fusion de données actimétriques pour la télésurveillance médicale. Projet de thèse, université Joseph Fourier.

[2] La telesante : un nouvel atout au service de notre bien-être : Un plan quinquennal écoresponsable pour le déploiement de la télésanté en France.(2009), Rapport remis à Madame Roselyne Bachelot-Narquin, Ministre de la Santé et des Sports par Monsieur Pierre Lasbordes, Député de l'Essonne.

[3] Agostino Giorgio. 2011. Innovative Medical Devices for Telemedicine Applications. In Telemedicine Techniques and Applications, Edited by Georgi Graschew and Stefan Rakowsky, pp 19-44.

[4] T. Gruber, 2003. Towards principals for the design of ontologies used for knowledge sharing. Formal ontology in conceptual analysis and knowledge representation. Kluwer Academic pubishers.

[5] Studer Benjamins Fensel. 1998. Knowledge Engeeneering : Principles and Methods. Data and Knowledge Engineering 25, 161-197

[6] J. Sowa. 1995. Top-level ontological categories. International Journal of Human and Computer Studies, 43, 669-685. 
[7] R. Mizoguchi, K. Kozaki, T. Sano et Y. Kitamura 2000. Construction and Deployment of a Plant Ontology. The 12th International Conference, EKAW2000, 113-128.

[8] A. Maedche. 2002. Ontology Learning for the Semantic Web. Boston: Kluwer Academic Publishers.

[9] C. Franco, J. Demongeot, C. Villemazet, N. Vuillerme. 2010. Behavioral telemonitoring of the elderly at home : Detection of nycthemeral rhythms drifts from location data. 24th International Conference on Advanced Information Networking and Applications Workshops, 759-766.

[10] Tareq Hadidi, Norbert Noury. 2009. A Predictive Analysis of the Night-Day Activities Level of Older Patient in a Health Smart Home. Ambient Assistive Health and Wellness Management in the Heart of the City. 290-293

[11] C. Franco, J. Demongeot, Y. Fouquet, C. Villemazet, N. Vuillerme. 2010. Perspectives in home TeleHealthCare system: Daily routine nycthemeral rhythm monitoring from location data. International Conference on Complex, Intelligent and Software Intensive Systems. 611-617.

[12] Anthony Fleury, Michel Vacher, Norbert Noury. 2010. SVM-Based Multi-Modal Classification of Activities of Daily Living in Health Smart Homes: Sensors, Algorithms and First Experimental Results. IEEE TRANSACTION ON INFORMATION TECHNOLOGY IN BIOMEDICINE. 274-283.

[13] Rifat Shahriyar, Md. Faizul Bari, Gourab Kundu, Sheikh Iqbal Ahamed, and Md. Mostofa Akbar. 2009. Intelligent Mobile Health Monitoring System (IMHMS). International Journal of Control and Automation, Vol.2, No.3, 13-28.

[14] Jean-Pierre Thomesse et l'équipe du projet TIISSAD. 2002. Les technologies de l'information intégrées aux services de soin à domicile : Le projet TIISSAD. Informatique et santé, 27-34.

[15] V. Stoicu-Tivadar, L. Stoicu-Tivadar, V.Topac and D. Berian. 2009. A WebServicebased Alarm Solution in a TeleCare System. 5th International Symposium on Applied Computational Intelligence and Informatics. 117-121.

[16] Aniello Minutolo, Giovanna Sannino, Massimo Esposito, Giuseppe De Pietro. 2010. A rule-based mHealth system for cardiac monitoring. 2010 IEEE EMBS Conference on Biomedical Engineering \& Sciences.

[17] Federica Paganelli, Dino Giuli. 2011. An Ontology-Based System for Context-Aware and Configurable Services to Support Home-Based Continuous Care. IEEE TRANSACTIONS ON INFORMATION TECHNOLOGY IN BIOMEDICINE, VOL. 15, NO. 2, MARCH 2011.

[18] Miguel A. Valero, Laura Vadillo, Iván Pau, and Ana Peñalver. 2009. An Intelligent Agents Reasoning Platform to Support Smart Home Telecare. IWANN 2009, Part II, LNCS 5518. 679-686.

[19] F. Latfi, B. Lefebvre and C. Descheneaux. 2007. Le rôle de l'ontologie de la tâche dans un Habitat Intelligent en Télé-Santé. 1ères Journées Francophones sur les Ontologies JFO, Sousse, Tunisie, 18 - 20 Octobre 2007.

[20] F. Latfi, C. Descheneaux, B. Lefebvre. 2007. Habitat intelligent en télé-Santé : ontologie de l'équipement. FICCDAT, 16-19 Juin. Toronto, Canada, 2007. 
[21] F. Latfi, B. Lefebvre, C. Descheneaux. 2007. Ontology-based management of the telehealth smart home, dedicated to elderly in loss of cognitive autonomy, OWLED 2007. June 6-7 Innsbruck, Austria.

[22] Rocio Martinez-Lopez, David Millan-Ruiz, Alberto Martin-Dominguez, Maria Aranzazu Toro-Escudero. 2008. An architecture for Next-generation of Telecare Systems using ontologies. Rules Engines and Data Mining.

[23] T.A Gavrilova, R.A Ravodin, E.S. Bolotnikova. 2011. Development of Dermatovenereology Ontology for Education and Expert Support.

[24] B.E. Dixon, A. Zafar, J.J. McGowan. (2007), Development of a Taxonomy for Health Information Technology, in K. A. Kuhn, J. R. Warren \& T.-Y. Leong, eds, ‘Proceedings of the 12th World Congress on Health (Medical) Informatics, MEDINFO 2007', Vol. 129 of Studies in Health Technology and Informatics, IOS Press, Brisbane, Australia, pp. 616-620. 